\title{
Telehealth Adoption: Three case studies at the organisational level
}

\section{Sophie Cockcroft}

School of Management and Enterprise

University of Southern Queensland

Springfield QLD Australia

Email: sophie.cockcroft@usq.edu.au

\section{Jane Hendy}

Brunel Business School

Brunel University, London

Email: jane.hendy@brunel.ac.uk

We present the results of an inductive analysis using interview data to establish core themes in organisational level adoption of telehealth. The source of the interview data was a large study to evaluate how telehealth can promote long term health and independence and improve quality of life for people suffering from chronic health conditions. The study took place over three different pilot sites and 41 interviews were conducted with healthcare professionals who collectively had responsibility for over 5000 patients. We explore the data from the perspective of the organisation, focussing on the unique features of each pilot site and seeking evidence for themes of adoption success at the organisational level, particularly via organisational identity. We explore the data via a set of propositions based on organisational identity theory and models of technology adoption. The major finding of this work is that experience in the adoption of telecare was found to detract from telehealth adoption success.

Keywords Teleheath, Telecare, Telemedicine, Organizational theory, Technology adoption 


\section{Introduction and definitions}

The present work is a qualitative study at the confluence of Information Systems (IS) and management. It is focussed on Healthcare at the organisational level. It explores how the nature of macro and meso levels (top and middle) in an organisation impact on technology adoption. Likewise in IS there are a preponderance of studies exploring the individual level of IS interaction, but less at the organisational level (Burton-Jones and Gallivan 2007). However, there has been an emergence of context specific theories, as opposed to general theories, surrounding adoption and use, which by their nature have a more organizational focus (Hong et al. 2013). Theories exist within IS that have evolved with a focus on external validity. That is the more general a theory is, the wider its applicability. Context specific theories are those which acknowledge that technology cannot be studied outside its social context. In particular such work explores how a network of affordances, i.e. factors that support use, lead to the achievement of organizational goals and how these affordances are put into action (Strong et al. 2014). The work presented here is specific to the context of telehealth adoption.

Telemedicine and telehealth have received some interest in the Information Systems literature but comparatively few papers explore organisational aspects (Al-Qirim 2007; Bower et al. 2011; Christensen et al. 2014; Elbanna and Linderoth 2015; Harrop 2001; Hu et al. 2002; LeRouge et al. 2012; Norum et al. 2007a; Norum et al. 2007b; Tarakci et al. 2009). A significant contribution to telemedicine research is the momentum project in Europe, deliverable 3.4 of which, published in 2016 (Jensen et al. 2016) reveals some findings in common with the present work. Which are discussed at the end of this paper.

We propose that one contributing factor in this research gap is that the organization has not been examined as an actor with agency in its own right with history and work practices that need to be accounted for. In this work we look at a unique set of cases in which the mandate for adoption came from the national level. By examining the history and culture (as expressed by organizational characteristics) of each of the three organizations and via a comprehensive set of interview data, we use inductive analysis to develop initial theoretical prepositions based on organizational theory, theory of effective use and models of technology adoption. We then perform a deductive analysis, following the approach of (Rivard et al. 2011) to test our propositions on the relationship between telehealth intervention and admissions, barriers to participation, experience of frontline health care professionals and organizational alignment. One interesting finding was that prior adoption experience was found to detract from adoption success. The major contributions of the work are first, the identification of themes that account for an organizational level of adoption and, second, the development of a model of organizational identity and processes and their impact on outcomes in technology adoption.

For the purposes of this study we adopt the following definitions of Telecare, Telehealth and telemedicine (Bower et al. 2011)

Telecare is the remote, automatic and passive monitoring of changes in an individual's condition or lifestyle (including emergencies) in order to manage the risks of independent living. Examples include movement sensors, falls sensors, and bed/chair occupancy sensors. These technologies are generally provided to patients with social care needs.

Telehealth describes a system that allows the remote exchange of data between a patient (at home) and health care professionals (at a Monitoring Centre) to assist in the management of an existing longterm condition(s) (Chronic obstructive pulmonary disease (COPD), diabetes, heart failure). The peripheral devices used by intervention participants in the Whole System Demonstrator (WSD) Telehealth Trial to monitor vital signs were tailored to their clinical needs and could include a blood pressure monitor, blood glucose monitor, blood oxygen monitor and weight scales

Telemedicine is the most comprehensive of the three definitions and is sometimes conceptualised as encompassing them. The WHO definition of telemedicine or e-health is, 'the practice of medical care using interactive audio-visual and data communications. This includes the delivery of medical care, diagnosis, consultation and treatment, as well as health education and the transfer of medical data'

\subsection{Research Question:}

How does organisational identity at the meso level impact on the adoption of innovations in the context of healthcare? 


\subsection{Background and Propositions}

King et al. (2010) highlighted the need to understand explicate and research the qualities of organizations as social actors. Organizations are typically portrayed as aggregations of individuals, or actors in a social network without agency in their own right. In an earlier study Albert and Whetten (1985) identified the features of an organizational identity as central, enduring, and distinctive/distinguishing (CED). A Central feature is one that has changed the history of the company; if this feature was missing, the history would have been different. Enduring features are deeply engrained in the organization; they have stood the test of time and are often explicitly considered sacrosanct or embedded in the organizational history. Distinguishing features are used by the organization to separate itself from other similar organizations, but can also set minimum standards and norms for that type of organization.

King et al also discussed the notion of identity evolving within organizations. Organizations in their early stages of existence are more amenable to change. Hendy, Chrysanthaki and Barlow (Hendy et al. 2012) draw on the existing literature describing this change process describing how in order to succeed organizations need to go through a process of identity transformation. With reference to Fiol (2002) they argue that it is conceptually incorrect to regard organizations as nothing more than aggregated human behaviour. It is emerging that the uniqueness of organizations as social actors has implications for organization level research.

From the IS perspective, Bélanger et al. (2014) explored multilevel research specifically in the IS context, of interest here is the research area identified, that rejects the reductionist, individualist view of IS evaluation in favour of relational variables of the group such as conflict cohesion confidence and trust. Specifically in terms of Health Information Technology (HIT), Rivard, Lapointe and Kappos (2011) identified conflicting imperatives in clinical IS implementation. And finally at a more general level (Venkatesh et al. 2003) sought organizational impacts on IS success. We contend that the implementation of health systems in particular demands organizational level evidence for the purposes of accountability and interpretation of government mandates. Based on the studies described above, we put forward the following propositions

P1 Organisations in earlier stages of technological maturity will be more likely to succeed in implementing telehealth innovations (King, Felin et al. 2010)

P2 Esteem/Kudos and confidence within organisations promote adoption (Belanger et al. 2014)

P3 The conflict of strategic imperatives across professional groups (cost reduction and care) impact the success of telehealth innovations (Rivard et al. 2011)

P4 organisational context for example leadership, technological literacy impact on success of telehealth innovations (Venkatesh et al. 2003)

\section{Description of the study}

This work is based on a large, UK government funded, evaluation program surrounding the UK Whole System Demonstrator Programme (WSD) which ran from 2008-2011 it was the largest multisite evaluation of telehealth technologies to date. The study as a whole involved 6191 patients and 238 general practitioner practices. The evaluation (subset of project described here) concerned implementation, impact and acceptability of telecare and telehealth (Chrysanthaki et al. 2013). It was structured as a randomised control trial (RCT) in which some participants used telehealth innovations and others a more traditional telecare model. In this work we focus on telehealth adoption, as this was the main policy driver for the work because it was novel (and thought be cost saving) but was also largely unknown and not adopted (unlike telecare which was already widely spread throughout the UK). Healthcare professionals were engaged in delivering Telehealth devices to the target segment of patients and educating them in their use. Telehealth concerns the more data driven technical aspects of telemedicine as distinct from telecare which derives from a focus on home care via telecommunications. In terms of site selection the originators of the study framed the trial as a large scale, pragmatic health technology assessment trial, designed to randomise suitably large numbers of patients and assess the impact of a broad class of telemonitoring technologies in the context of routine delivery of NHS care. The impetus for this study was policy driven via the UK department of health. 
In accomplishing the roll out of the new systems the Department of Health (DH) and local level organizations had very different interpretations of policy and goals and implementation process. We develop the concepts around this, and an analytical framework via formal brainstorming techniques and a modified grounded theory approach (Gioia et al. 2013). This research process is known as analytic induction.

The three sites in our study show contrasting characteristics; the first is an urban London borough with high density housing and culturally diverse population. The remaining two cases concern rural areas and demonstrate challenges more associated with remoteness, lack of public transport and an ageing population. The three organizations also had key differences in management approach. Differences are evident in terms of management styles and positioning of power, autonomy of professionals, and strategic alignment of cost of care and clinical effectiveness. The expected pace of implementation also differed. In particular, the sites all had the same project deadlines but their implementation delivery time differed. The rhetoric between sites differed quite markedly in terms of how they viewed the effective use of technology and how they understood success. In particular there is some conflict between Local authorities who traditionally manage social care and primary care trusts (PCTs) who take a more medicalised view. PCTs were largely administrative bodies, responsible for commissioning primary, community and secondary health services from providers. Since the study PCTs have been abolished in favour of clinical commissioning groups.

\subsection{Case 1 Newham}

Newham is characterised as an urban, low socio-economic area with a large volume social housing. Public transport links are good. In Newham the organizational lead came from the Local Authority (LA), the London borough of Newham. They had a previous successful telecare service and there had been two minor telehealth trials one for diabetes and one for antenatal care. The money to run this service came from the social care budget of the borough council. They had a project champion in the form of the Mayor who made an early and significant investment in telecare. The project carried a lot of Kudos in the local community. In the transition to the WSD and the more medical approach of telehealth there were some challenges in communication between the LA and the Primary care trust (PCT). Health and social care, but they were encouraged by early success in this integration and winning the WSD bid to conduct the trial in Newham. There were expectations from the borough about large financial savings. The PCT and the LA which were attempting to integrate had separate IS and Finance departments which made the level of integration needed for WSD difficult. The ethnic diversity in the borough lead to some difficulties with recruiting participants for the RCT. In adopting telehealth Newham started with new technology from Philips with which the LA was not familiar.

\section{$2.2 \quad$ Case 2 Kent}

Kent is a rural community, with poor public transport links, and mixed employment opportunities. In Kent the organizational lead also came from the local authority. It is the largest local authority in England with 1.3 million people at the time of the study. They had a project champion in the form of a general practitioner (GP) but that person was in a minority amongst their profession. Many GPs felt alienated by the project and saw it as another overhead on their time. It was, however embraced by the community matrons. There was a high level of enthusiasm in phase I, but it waned in phase II. IT literacy among staff was assisted by the fact that they used the same telehealth provider (Viterion) as they had done in a prior successful trial (pre 2008). Kent had three primary care trusts, and this provided even greater communication challenges than Newham. In accomplishing the roll out of the new systems the Department of Health (DH) and local level organizations had very different interpretations of policy and goals and implementation process.

\subsection{Case 3 Cornwall}

Cornwall is a coastal and rural community with some isolated communities and low employment rates. In Cornwall the organizational lead came from the PCT. There was a Local administration team Cornwall NHS based in a central office, and TH monitoring nurses and Community matrons were attached to various GP surgeries throughout Cornwall. Cornwall had a delayed start due to a change in the choice of technology. However, when they were up and running, they had a quicker implementation. Cornwall started lower than the other two WSD sites in the sense that there had been no prior trials. In addition to organizational lead from the PCT they employed a management team to oversee the 
implementation. WSD introduced a culture of competition between the sites, and while there was little time or opportunities to share knowledge and experience, in this case there was some discussion between West Kent and Cornwall which resulted in them taking up some of the same technology.

\section{Methodology}

Interviews were conducted, by experts, with stakeholders at all levels in the organization the study took place over three different pilot sites and 41 interviews were conducted with healthcare professionals and others who collectively had responsibility for over 5000 patients. The interviewees comprised 10 Local authority managers, 14 National Health Service Managers and 17 other associated staff. The interview data was analysed using a modified grounded theory approach described below.

(Gioia et al. 2013) provide guidelines for analysing interview data into first and second-order categories and developing these categories into a more structured form known as a data structure (see Figure 1). The data structure was developed via a brain storming session, and using the interview data. In the firstorder analysis, interviewees' figures of speech were used and there was little attempt to develop explicit categories for open coding in a similar fashion to that proposed by Strauss and Corbin (2004). In the process advocated by Strauss and Corbin, where there is not a direct quote that can be drawn from the interview transcripts (yet a construct clearly exists) a short descriptive phrase is developed. In the second-order analysis, the authors extracted similarities and differences between these categories (i.e. we engaged in what Strauss and Corbin (2004) term axial coding) in order to develop the 1st order codes into themes. Finally, we aggregated similar second-order themes into five dimensions that make up the basis of the emergent data structure. Whilst no implicit relationship between second order factors is captured, this data analysis approach allows 'a recursive process-oriented, analytic procedure that continued until we firmly grasp the emerging theoretical relationships and additional interviews failed to reveal new data relationships' (Lapointe and Rivard 2005 p. 183). By this means we identified tensions, struggles, and fractures, at the intersection between local and DH policy.

\section{Results}

The interview data is encapsulated and summarised in the Gioia data structure (Figure 1) which tells the story of telehealth adoption at the three sites, and the common themes emerging. Generally organisations reacted to external policies/triggers from the department of health (left side of Figure 1), by drawing on identity, specific skills and expertise, and IT capabilities and Infrastructure. These would then trigger a search for meaning within the professional groups within the organisation, often engendering some misalignment, which impacted the success or otherwise of the telehealth delivery. This analysis reveals dynamic relationship around the intersection of policy at the UK government Department of health (DH - macro) and local (organizational - meso) level sometimes referred to as the level of the hive (Abrahams 2002), and how the organization as actor with identity, agency, history, context and local policy impacts on the adoption of innovation. In particular the effects of external factors (e.g. changes in government, and meso level tensions emanating from leadership and "followership" and the alignment of strategic objectives at different levels). That is, both changes in government at a national level and changes at a local level such as a change in the director of a local authority are likely to be the source of external factors. Followership is quality demonstrated by employees within an organization typified by a willingness to accept direction and guidance from leaders recognising that any effective organization needs both leaders and followers. The triggers for implementation at the macro level, came from a need to develop efficient ways of managing people with long term conditions. Some impetus for joining the study on behalf of the sites studied came from the kudos of being an early adopter. As the project progressed fractures emerged at various levels. In particular some professionals felt their autonomy was being taken away when they had to put aside patient care in favour of the RCT - for example not offering telehealth style monitoring to someone who could benefit from it. Or similarly, when pressure was felt to pursue cost saving over patient well-being. Thus the shared understanding of the meaning of care and what constituted success of the project was lost as the project progressed. In Newham the level of political connectedness to the macro or DH level was more pronounced with the presence of a Mayor who championed the project. In terms of WSD management structures, Cornwall emerged as an unexpected success story, having comparatively less telecare experience of IT infrastructure, the participants accepted the deployment of a management team to oversee the implementation. 


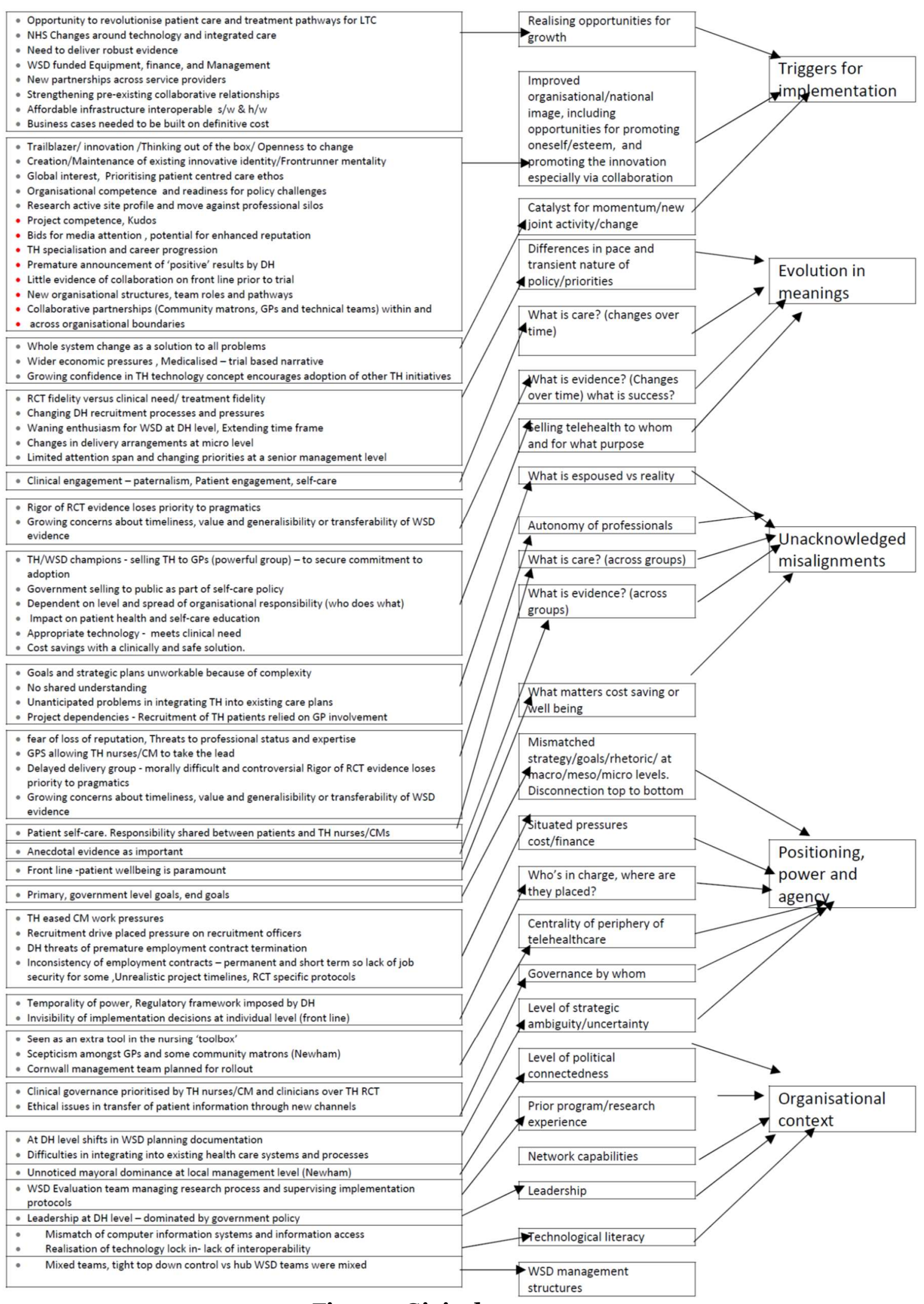

Figure 1 Gioia data structure 


\section{Discussion}

In the early phase, the project managers wanted to present image of competence and were often very keen to go mainstream before they got the results of the trial and further. In addition the pace of the project was dictated by the $\mathrm{DH}$ and, in contrast at the local level, there was more emphasis on getting things right. Whilst the pressure of this permeated down it did not affect those at the bottom level. Between these two levels there was a switch between the priority being cost and the priority of wellbeing. This phenomenon was at the core of the mismatch in strategy as the policies permeated down.

GPs had enormous power and in some cases changed how the randomised control trial was run. In some cases interviewees suggested that GPS put up obstacles. Both nurses and doctors had monitoring schemes but the Doctors did not want to get involved with using them. Nurses were often on short term contracts. Many professionals felt that they had power in recruitment but not in delivery. On the flip side there was (anecdotally some power that was not visible in the form of nurses "rebelling" and deciding who should have telehealth). There was power in relation to governance National Institute for Health and Care Excellence (NICE) guidelines were promulgated from above but levels of accountability seemed to differ between sites. Some sites were more politically connected, for example Newham had the mayor on board. Cornwall did not have strategic certainty but their power derived from the fact that they employed a specialist management team to oversee the Nurses and equipment. Early analysis suggests that this qualitative study provides some support for theory from traditional IS adoption literature, but further analysis is required to provide pointers to the nature of mid-range context specific theory needed to explore the meso-level constructs unearthed in this work. In a general sense these findings overlap with the Key Performance Indicators identified by Jensen et al (2016) in the Momentum study. Specifically in regard to cultural readiness, the presence of a champion, and change management planning. 


\section{References}

Abrahams, M. 2002. "The Beehive of Organisational Excellence: Entrenching Workplace Practices That Lead to High Performance." Stellenbosch: Stellenbosch University.

Al-Qirim, N. 2007. "Championing Telemedicine Adoption and Utilization in Healthcare Organizations in New Zealand," International Journal of Medical Informatics (76:1), pp. 42-54.

Albert, S., and Whetten, D.A. 1985. "Organizational Identity," Research in organizational behavior).

Belanger, F., Cefaratti, M., Carte, T., and Markham, S.E. 2014. "Multilevel Research in Information Systems: Concepts, Strategies, Problems, and Pitfalls," Journal of the Association for Information Systems (15:9), pp. 614-650.

Bélanger, F., Cefaratti, M., Carte, T., and Markham, S.E. 2014. "Multilevel Research in Information Systems: Concepts, Strategies, Problems, and Pitfalls," Journal of the Association for Information Systems (15:9), p. 614.

Bower, P., Cartwright, M., Hirani, S.P., Barlow, J., Hendy, J., Knapp, M., Henderson, C., Rogers, A., Sanders, C., Bardsley, M., Steventon, A., Fitzpatrick, R., Doll, H., and Newman, S. 2011. "A Comprehensive Evaluation of the Impact of Telemonitoring in Patients with Long-Term Conditions and Social Care Needs: Protocol for the Whole Systems Demonstrator Cluster Randomised Trial," BMC Health Services Research (11:1), p. 184.

Burton-Jones, A., \& Gallivan, M. J. 2007. Toward a deeper understanding of system usage in organizations: a multilevel perspective. MIS quarterly, pp 657-679.

Christensen, H.B., Hansen, K.M., Kyng, M., and Manikas, K. 2014. "Analysis and Design of Software Ecosystem Architectures - Towards the 4s Telemedicine Ecosystem," Information and Software Technology (56:11), pp. 1476-1492.

Chrysanthaki, T., Hendy, J., and Barlow, J. 2013. "Stimulating Whole System Redesign: Lessons from an Organizational Analysis of the Whole System Demonstrator Programme," Journal of Health Services Research \& Policy (18), pp. 47-55.

Corley, K.G., and Gioia, D.A. 2004. "Identity Ambiguity and Change in the Wake of a Corporate SpinOff," Administrative Science Quarterly (49:2), pp. 173-208.

Elbanna, A., and Linderoth, H.C.J. 2015. "The Formation of Technology Mental Models: The Case of Voluntary Use of Technology in Organizational Setting," Information Systems Frontiers (17:1), pp. 95-108.

Fiol, C.M. 2002. "Capitalizing on Paradox: The Role of Language in Transforming Organizational Identities," Organization Science (13:6), pp. 653-666.

Gagnon, M.P., Simonyan, D., Ghandour, E.K., Godin, G., Labrecque, M., Ouimet, M., and Rousseau, M. 2016. "Factors Influencing Electronic Health Record Adoption by Physicians: A Multilevel Analysis," International Journal of Information Management (36:3), pp. 258-270.

Gioia, D.A., Corley, K.G., and Hamilton, A.L. 2013. "Seeking Qualitative Rigor in Inductive Research: Notes on the Gioia Methodology," Organizational Research Methods (16:1), pp. 15-31.

Harrop, V.M. 2001. "Virtual Healthcare Delivery: Defined, Modeled, and Predictive Barriers to Implementation Identified," Journal of the American Medical Informatics Association), pp. 244-248.

Hendy, J., Chrysanthaki, T., Barlow, J., Knapp, M., Rogers, A., Sanders, C., Bower, P., Bowen, R., Fitzpatrick, R., and Bardsley, M. 2012. "An Organisational Analysis of the Implementation of Telecare and Telehealth: The Whole Systems Demonstrator," BMC health services research (12:1), p. 1.

Hong, W., Chan, F.K., Thong, J.Y., Chasalow, L.C., and Dhillon, G. 2013. "A Framework and Guidelines for Context-Specific Theorizing in Information Systems Research," Information Systems Research (25:1), pp. 111-136.

Hu, P.J.H., Chau, P.Y.K., and Sheng, O.R.L. 2002. "Adoption of Telemedicine Technology by Health Care Organizations: An Exploratory Study," Journal of Organizational Computing and Electronic Commerce (12:3), pp. 197-221.

Jensen, L.K., Knarvik, U., Pedersen, C.D., Wenche, T., and Whitehouse, D. 2016. "Deliverable 3.4 Personalised Blueprint for Telemedicine Deployment: Validated and Tested Version."

King, B.G., Felin, T., and Whetten, D.A. 2010. "Perspective-Finding the Organization in Organizational Theory: A Meta-Theory of the Organization as a Social Actor," Organization Science (21:1), pp. 290305 .

Lapointe, L., and Rivard, S. 2005. "A Multilevel Model of Resistance to Information Technology Implementation," MIS quarterly), pp. 461-491.

LeRouge, C., Garfield, M.J., and Collins, R.W. 2012. "Telemedicine: Technology Mediated Service Relationship, Encounter, or Something Else?," International Journal of Medical Informatics (81:9), pp. 622-636. 
Norum, J., Pedersen, S., Størmer, J., Rumpsfeld, M., Stormo, A., Jamissen, N., Sunde, H., Ingebrigtsen, T., and Larsen, M.-L. 2007a. "Prioritisation of Telemedicine Services for Large Scale Implementation in Norway," Journal of telemedicine and telecare (13:4), pp. 185-192.

Rivard, S., Lapointe, L., and Kappos, A. 2011. "An Organizational Culture-Based Theory of Clinical Information Systems Implementation in Hospitals," Journal of the Association for Information Systems (12:2), p. 123.

Strong, D.M., Volkoff, O., Johnson, S.A., Pelletier, L.R., Tulu, B., Bar-On, I., Trudel, J., and Garber, L. 2014. "A Theory of Organization-Ehr Affordance Actualization," Journal of the Association for Information Systems (15:2), pp. 53-85.

Tarakci, H., Ozdemir, Z., and Sharafali, M. 2009. "On the Staffing Policy and Technology Investment in a Specialty Hospital Offering Telemedicine," Decision Support Systems (46:2), pp. 468-480.

Venkatesh, V., Morris, M.G., Davis, G.B., and Davis, F.D. 2003. "User Acceptance of Information Technology: Toward a Unified View," MIS quarterly), pp. 425-478.

\section{Copyright}

Copyright: (C) 2018 Cockcroft \& Hendy. This is an open-access article distributed under the terms of the Creative Commons Attribution-NonCommercial 3.0 Australia License, which permits noncommercial use, distribution, and reproduction in any medium, provided the original author and ACIS are credited. 